\title{
Validación de un modelo explicativo del proceso de envejecer con éxito a partir de aspectos psicológicos, físicos, relacionales y de ocio
}

\author{
Amparo Oliver ${ }^{1}$, Melchor Gutiérrez ${ }^{1}$, José M. Tomás ${ }^{1}$, Laura Galiana ${ }^{1}$ \\ y Patricia Sancho ${ }^{2}$ \\ ${ }^{1}$ Universitat de València (España); ${ }^{2}$ Universidad de Zaragoza (España)
}

\begin{abstract}
Cada vez más, la investigación sobre el proceso de envejecimiento tiende a adoptar una doble perspectiva emic (específico a una cultura) y etic (universal), necesitándose más estudios que atiendan un enfoque transcultural. El objetivo es obtener respuesta empírica a la pregunta de si un modelo de envejecimiento con éxito planteado desde un contexto diferente como es el taiwanés, es adecuado para representar este proceso en nuestros mayores. La muestra estuvo formada por 737 personas mayores. Se recabaron datos de variables socio-demográficas y diversas escalas para evaluar satisfacción con la vida, ocio, salud y apoyo social. Se puso a prueba un modelo de ecuaciones estructurales tomado de Lee et al. (2011), en el que ocio, salud y apoyo social predecían satisfacción con la vida. Los resultados obtenidos mostraron un ajuste adecuado. En concreto, se observó que, en el marco de otros factores que teóricamente influyentes -salud y apoyo social-, este novedoso aspecto sobre cómo las personas mayores gestionan su tiempo de ocio, aunque explicativo, no es un factor tan determinante como en el estudio de referencia.
\end{abstract}

Palabras clave: Envejecimiento con éxito, apoyo social, ocio, satisfacción con la vida, modelos de ecuaciones estructurales.

Validation of an explicative model of the successful aging process with psychological, physical, relational, and leisure variables: Increasingly, research on the aging process tends to adopt a dual perspective emic (specific to a culture) and etic (universal), requiring further studies to address this cross-cultural approach. The aim is to obtain an empirical answer to the question of whether a model of successful aging raised from a different context such as the Taiwanese context, is suitable to represent this process in our elders. The sample consisted of 737 elderly. Socio-demographic-data and different scales to assess life satisfaction, leisure, health, and social support were collected. A structural equation model taken from Lee et al. (2011), in which leisure, health and social support predicted life satisfaction, was tested. Results showed a proper fit. In particular, it was observed that, in the context of other factors theoretically influential -health and social support-, this new aspect of how older people manage their leisure time, although explanatory, is not such a determining factor as in the study of reference.

Keywords: Successful aging, health, social support, leisure, life satisfaction, structural equation modeling.

Correspondencia: Amparo Oliver. Departamento de Metodología de las Ciencias del Comportamiento. Universitat de València. Av. Blasco Ibáñez, 21. C.P.: 46010, Valencia. (España). E-mail: oliver@uv.es 
El envejecimiento, como cualquier proceso que vive cada individuo en sociedad, es susceptible de ser estudiado desde una perspectiva que atienda a su contexto. Pike (1954), acuñando los términos emic y etic, en clara analogía a la fonémica y la fonética, fue el primero en llamar la atención sobre la importancia de investigar desde una perspectiva particularmente adscrita a una cultura, a un dominio, o a un ámbito más general.

Cada vez más, queda constancia de que la investigación sobre el proceso de envejecimiento ha de adoptar también esta doble perspectiva emic (específico a una cultura) y etic (universal) porque existe poca investigación comparativa, y se necesiten más estudios que atiendan o planteen un enfoque transcultural (Alegría et al., 2009). Efectivamente, cuando se revisa la literatura científica en investigación cualitativa en envejecimiento con éxito, se pone de manifiesto la baja representatividad que existe de estudios procedentes de países no occidentales, así como la homogeneidad cultural de los participantes en las investigaciones (Cosco, Prina, Perales, Stephan, y Brayne, 2013). Recientemente, algunas investigaciones apuntan hacia estos objetivos y desarrollan investigaciones paradigmáticas en este terreno que, a pesar de las dudas sobre la representatividad de las muestras por su reducido tamaño y el tipo de muestreo llevado a cabo, que pueden captar el interés del lector y resultar muy estimulantes (Karlin, Weil, Saratapun, Pupanead, y Kgosidialwa, 2014). Por ejemplo, Hilton, González, Saleh, Maitoza, y Cole (2012) investigan en una muestra con latinos en Estados Unidos que, a pesar de ser la minoría con mayor y más rápido crecimiento, han sido ignorados en la literatura científica sobre investigación en envejecimiento de éxito. Estos autores, entre otras medidas, usaron cuestionarios validados como el de Phelan de envejecimiento con éxito, para conocer si era capaz de captar las propias percepciones de 60 latinos residentes en tres diferentes estados del oeste norteamericano. Al comparar los resultados con aquellos procedentes del ámbito anglosajón, japonés, japonés-americano y latinoamericano, encontraron que latinos y latinoamericanos daban respuestas muy similares, compartían una visión más parecida del envejecimiento de éxito y se distanciaban de los otros grupos culturales. Los latinos (la minoría latinoamericana residente en Estados Unidos) primaba el optimismo, la visión positiva y el vivir el presente, disfrutando su vida en comunidad, fomentando relaciones sociales y descansando en su espiritualidad y en su familia para encontrar alivio y significado a su edad, aunque la preocupación por la seguridad económica siempre se mantuviera presente. La investigación de Jane Hilton y colaboradores (2012), resulta también de interés porque da pautas para investigar en el ámbito, incluyendo los hitos a superar metodológicamente y los debates que se mantienen abiertos en tópicos de interés en la literatura científica actual sobre envejecimiento con éxito.

Pero trabajemos en modo emic o etic, si nos centramos en el envejecimiento con éxito o positivo, óptimo, saludable, activo, productivo o cualquiera de sus 
denominaciones (Fernández-Ballesteros et al., 2008), entenderemos que, por su multidimensionalidad, son numerosos los acercamientos empíricos posibles. Por ejemplo, existen varios modelos que podríamos tomar como referentes de etic y analizarlos desde una comparativa de perspectiva universal frente a específica a una cultura, en nuestro caso la española. Desde los modelos originales ya clásicos y con enorme repercusión en la literatura científica en psicogerontología de Rowe y Khan (1987) hasta conceptualizaciones y adaptaciones más recientes como los modelos de envejecimiento armónico de Liang y Luo (2012), el de Iwamasa e Iwasaki (2011), el de Arias e Iglesias (2015) o el de cuatro factores de Lee, Lan, y Yen (2011), varias aproximaciones son susceptibles de someterse a prueba empírica en cuanto a su validez de generalización a nuestro contexto.

Además, está la cuestión del interés por estudiar el contexto español, y en las siguientes líneas se profundiza en porqué es un escenario idóneo donde verificar si modelos teóricos que se han planteado en otros contextos intentando ser universales, tienen apoyo o evidencia empírica basada en datos de personas mayores en España. Como recientemente señalan Serrano, Latorre, y Gratz (2014), nuestro país es uno de los países europeos que ha vivido más cambios en el siglo XXI, en cuanto al aumento de la población envejecida. La mayor expectativa de vida, unida a la menor tasa de fertilidad provoca una ratio con más magnitud de personas mayores. Este cambio demográfico, como estos mismos autores señalan, implica desafíos en la calidad de vida de individuos y familias, así como en la economía y en la reorganización de los sistemas de prestación de los servicios sociales y de salud. Por esta razón es necesario avanzar en el conocimiento de los procesos que regulan este envejecimiento y lo llevan a ser vividos o no, de forma más exitosa, saludable o activa.

En definitiva, con el presente estudio, conocer la importancia relativa de los cuatro factores que Lee y colaboradores acotaran: psicológicos, físicos, de apoyo social y ocio activo sobre el envejecimiento con éxito. Este trabajo aborda una primera validación del modelo de Envejecimiento con Éxito basado en cuatro factores de Lee et al. (2011), planteado y validado originalmente y de forma reciente en contexto asiático según se representa en la gráfica 1. Esta aproximación está sólidamente fundada en los planteamientos teóricos de Baltes y Baltes (1990), o Rowe y Kahn (1987), entre otros enfoques. Se trata de una aproximación integral y parsimoniosa a través de factores psicológicos, físicos, de apoyo social y ocio activo como aspectos relevantes en la percepción de un envejecimiento activo, saludable o exitoso.

La gestión adecuada del tiempo de ocio es clave en otras etapas del ciclo vital como la adolescencia y juventud, en su conexión con el riesgo de exclusión social. Se trata ahora de ver cuál es su importancia relativa en el contexto de otros tres factores que se prevén relevantes en otra etapa clave del ciclo vital: el envejecimiento. En este ámbito concreto de investigación, el ocio debe entenderse más allá de una simple actividad en el 
tiempo libre, no restringirlo a su práctica, ni al denominado ocio serio (Brown, McGuire, y Voelkl, 2008), sino al valor experiencial que como señalan Lazcano, Madariaga, y Doistua (2010), subyace al ocio humanista y tiene incidencia en la calidad de vida de las personas y se asume entre las condiciones necesarias para un envejecimiento satisfactorio (Cuenca, 2006, 2012; Gañán y Villafruela, 2015).

Gráfica 1. Modelo teórico de envejecimiento con éxito basado en cuatro factores según Lee et al. (2011)

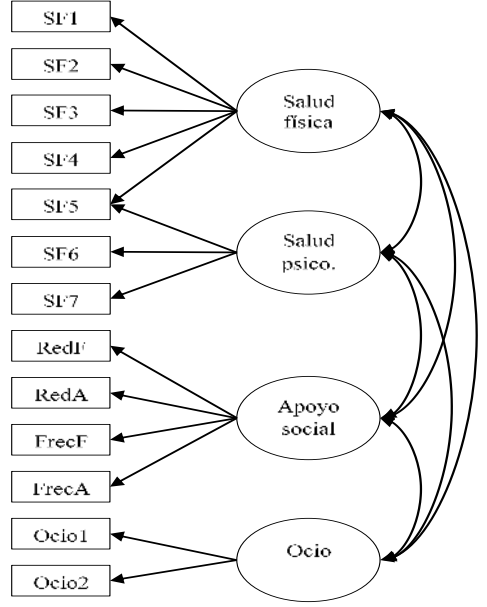

Nota: Para mayor claridad, no se muestran los errores estándar. Abreviaturas del modelo: SF (ítems de salud física); RedF (indicador de la red familiar); RedA (indicador de la red de amigos); FrecF (frecuencia de contacto familiar); FrecA (frecuencia de contacto con amigos); Ocio 1 y 2 (indicadores de actividad de ocio).

Así, el objetivo del presente artículo es obtener respuesta empírica a la pregunta de si un modelo de envejecimiento con éxito basado en cuatro grandes factores planteado desde un contexto tan diferente al nuestro como es el taiwanés, es adecuado para representar este proceso en nuestros mayores.

\section{MÉTODO}

\section{Participantes}

La tasa de respuesta fue del 77.54\%. La muestra estuvo formada por 737 personas de 55 años de edad o mayores con edad media de 65.41 años $(D T=6.6)$, desde 55 a 92 años. El 69\% fueron mujeres. En cuanto al nivel de estudios, el 28.4\% contaban con estudios primarios, un $41.9 \%$ con estudios secundarios y un $29.7 \%$ con estudios superiores. Finalmente, y con respecto al estado civil, el $67.5 \%$ estaban casados, un $15.7 \%$ eran viudos, un $10 \%$ solteros y un $6.7 \%$ divorciados. 


\section{Instrumentos}

Por lo que respecta a esta investigación concreta, se recabaron datos de variables socio-demográficas y diversas escalas para evaluar variables de tipo sociológico y psicológico. En concreto:

a) Escala de Satisfacción con la Vida (Diener, Emmons, Larsen, y Griffin, 1985). Instrumento formado por 5 ítems. Las puntuaciones se recogen en una escala tipo Likert con 5 anclajes, desde 1 (totalmente en desacuerdo) a 5 (totalmente de acuerdo).

b) Indicadores de ocio (Lee et al., 2011). La definición operacional fue la frecuencia en que se implican en actividades o ejercicio intenso y la frecuencia con que en los últimos 12 meses han salido de viaje fuera de su ciudad. Las respuestas podían variar de 1 (nunca) a hasta 6 (cinco veces o más).

c) Escala SF-8 de Salud (Ware, Kosinski, Dewey, y Gandek, 2001). Esta escala evalúa la salud relacionada con la calidad de vida, mediante 8 ítems que puntúan en una escala tipo Likert con 5 anclajes de respuesta. Es una versión de uso menos extendido de la de 36 ítems desarrollada por el mismo equipo (Ware, Snow, Kosinski, y Gandek, 1993), pero con características y comportamiento similar.

d) Escala de apoyo (Lubben y Gironda, 2004), mediante cuestionario de autoinforme de 12 ítems con cinco anclajes de respuesta para evaluar el tamaño, la cercanía y la frecuencia de contactos de la red social de las personas mayores. La mitad de los ítems preguntan sobre apoyo de los familiares, y los seis restantes sobre apoyo de amigos.

\section{Procedimiento}

La investigación tiene un diseño de panel, en el que participaron personas que acudían a los programas de aprendizaje a través del ciclo vital de la Universitat de València (Aulas de la Tercera Edad y Unimajors) durante el curso 2014-2015. En este trabajo, se utilizaron los datos recogidos en la primera ola del diseño. Se invitó a participar a todos los participantes en el programa. Las encuestas tuvieron lugar en las propias aulas, durante sesiones de aproximadamente 30 minutos de duración. Aunque el procedimiento era de papel y lápiz, se contó en todo momento con la presencia de investigadores y entrevistadores entrenados para solucionar posibles dudas que surgiesen.

\section{Análisis de datos}

La adecuación del modelo teórico desarrollado por Lee y colaboradores (2011), originalmente en contexto asiático, fue puesto a prueba en cuanto a su traslación al contexto español mediante un modelo de ecuaciones estructurales, en concreto, un modelo de estructuras de covarianza con variables observables e inobservables o factores. Las variables directamente observables fueron los ítems que se han descrito en 
la sección de instrumentos, y los factores son los cuatro constructos postulados por Lee et al. (2011) y que se asumen intercorrelacionados entre sí, además de un factor global de satisfacción con la vida que se pretendía explicar como acercamiento al envejecimiento con éxito.

El ajuste del modelo se evaluó mediante diversos criterios como recomienda la literatura: 1) Estadístico chi-cuadrado; 2) Comparative Fit Index (CFI; Bentler, 1990), con valores indicativos de buen de .90 o incluso de .95 según Hu y Bentler (1999) indicativos de un buen ajuste; y 3) el Root Mean Squared Error of Approximation (RMSEA; Steiger y Lind, 1980), con valores de .08 o menores como indicativos de un ajuste adecuado. El método de estimación empleado fue máxima verosimilitud con correcciones robustas para los errores estándar dada la naturaleza ordinal y no normal de los datos. Los datos se procesaron en SPSS 21 y posteriormente el modelo de ecuaciones estructurales se puso a prueba con Mplus 6.11.

\section{RESULTADOS}

\section{Ajuste del modelo}

Los resultados obtenidos fueron claramente indicativos de ajuste muy adecuado, $\chi^{2}(123)=479.635(p<.001)$, CFI $=.983 ;$ TLI $=.979 ;$ RMSEA $=.063$ [intervalo de confianza al 95\% .057-.069]. Se alcanzó un grado de explicación de un $20 \%$ de la satisfacción con los cuatro factores implicados.

Tabla 1. Correlaciones entre las dimensiones del envejecimiento con éxito

\begin{tabular}{lcccc}
\hline & Salud física & Salud psicológica & Apoyo social & Ocio \\
\hline Salud física & -- & & & \\
\hline Salud psicológica & $.61^{* *}$ & -- & & \\
\hline Apoyo social & .00 & $.22^{* *}$ & -- & \\
\hline Ocio & $.30^{* *}$ & $.20^{* *}$ & $.36^{* *}$ & -- \\
\hline Nota: ${ }^{* *}=p<.01$. & & &
\end{tabular}

El mapa de relaciones bivariadas, información obviada en el modelo y presentada para mayor claridad en la tabla 1, una vez más nos revela una elevada correlación (.61) entre salud física y psíquica, y nos muestra relación positiva y moderada entre ocio y apoyo social, pero sin embargo nula entre salud física y apoyo o red social. Este último resultado de especial interés aplicado. 
Gráfica 2. Resultados de estimaciones de coeficientes en puesta a prueba del modelo de envejecimiento con éxito prediciendo satisfacción con la vida acorde a planteamientos de Lee et al. (2011)

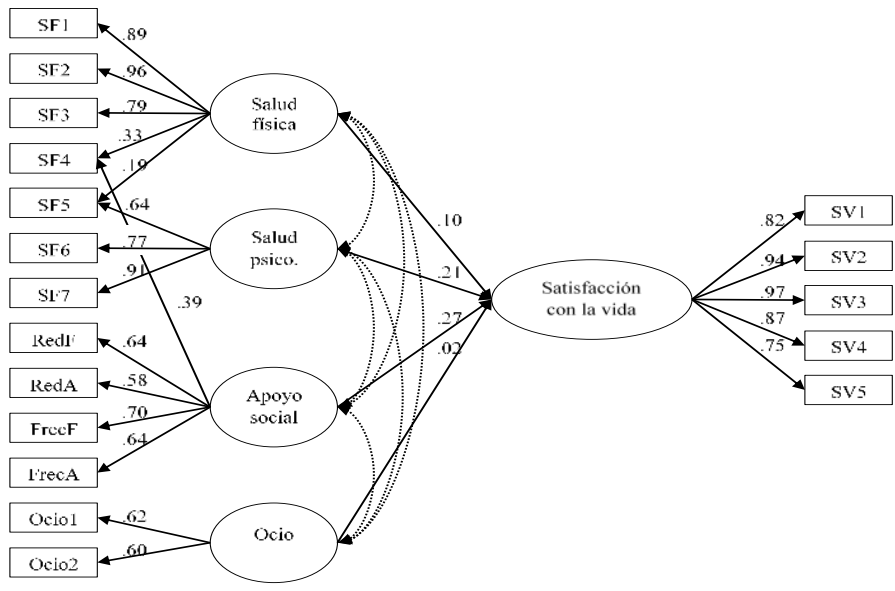

Nota: Para mayor claridad, no se muestran los errores estándar. El valor de las correlaciones entre las dimensiones del envejecimiento con éxito puede consultarse en la tabla 1.

\section{DISCUSIÓN Y CONCLUSIONES}

Alegria et al. (2009) pusieron de manifiesto la necesidad de considerar una perspectiva emic, al trabajar la equivalencia de las medidas e instrumentos en que se basan nuestras investigaciones y valorar con cuidado su capacidad de generalización cuando las evaluamos desde una perspectiva de etic, cuando en nuestro caso lo observamos en modelos universales o más generalistas. Con el interés en estos aspectos, investigadores e investigadoras, debemos ser capaces de mantener ese sutil equilibrio entre las perspectivas emic y etic también en el ámbito de estudio del envejecimiento con éxito.

La literatura sobre envejecimiento ya había puesto de relieve extensamente la importancia de las relaciones sociales (Baltes y Baltes, 1990; Rowe y Khan, 1987) y, efectivamente, el modelo respalda esta conclusión, puesto que las relaciones de mayor cuantía corresponden al apoyo social. Esto es así y con mayor intensidad que los componentes de salud física, al igual que encontraron recientemente Cosco et al. (2013). En cuanto al papel del ocio, los resultados del modelo no suponen una evidencia tan rotunda como resultados existentes en la literatura, que señalan que envejecientes con éxito participan una vez y media más en actividades de ocio de lo que lo hacen el resto de envejecientes (Chaves, Camozzato, Eizirik, y Kaye, 2009). En nuestro estudio, el efecto del ocio, aunque fuerte y positivamente relacionado con los otros factores clave del envejecimiento con éxito, apenas explica la satisfacción. 
Finalmente, en cuanto a su ajuste global, los resultados basados en el modelo avalan la generalización del modelo original de Lee y colaboradores (2011) surgido en contexto taiwanés al ámbito español. Respecto al ajuste analítico, al observar la prevalencia en el modelo de cada uno de los cuatro grandes factores, hemos de tener en mente los resultados de partida obtenidos por procedimientos estadísticos equivalentes en la investigación llevada a cabo por Lee et al. (2011) sobre 312 adultos taiwaneses mayores de 65 años. De mayor a menor peso en la definición empírica del constructo envejecimiento con éxito, los factores serían: físico (.91), psicológico (.88), ocio (.64) y por último lo social (.24).

Así, en el marco de otros factores que ya se asumían teóricamente influyentes -como el estado de salud física y psíquica y por supuesto el apoyo y la participación social-, concluimos que este novedoso aspecto sobre cómo las personas mayores gestionan su tiempo y actividades de ocio y placer aunque explicativo, no es un factor tan determinante como en el estudio de referencia, y por tanto, mientras no se replique en un mayor número de estudios en diferentes contextos, debe interpretarse en clave emic y no etic.

En cuanto a futuras líneas de investigación queda planteada la cuestión de conocer la versatilidad de estos modelos, su capacidad de generalización más allá de nuestro país, pensando en un contexto próximo en referencia a una lengua común sobre lo que existen investigaciones de referencia (Hilton et al., 2012) y el aproximarnos a la validez de estos modelos al contexto europeo comparando las similitudes y diferencias. El modelo explicativo de envejecimiento con éxito de Lee para el que hemos encontrado apoyo suficiente en los datos, es ya un buen punto de partida, entre otros aspectos con el hemos encontrado apoyo a la cita latina de Juvenal “... mens sana in corpore sano". Pero también ha aparecido un nuevo matiz, el de la desigual importancia que el ocio tiene en españoles y taiwaneses. Explicar la satisfacción de las personas mayores es un objetivo de primera magnitud, pues contribuir a que envejezcan satisfactoriamente no es solo importante para su propia calidad de vida, sino para la de sus familias y la sociedad (Kahng, 2008).

También incorporar nuevas variables al modelo como la generatividad que recientemente en nuestro contexto Arias e Iglesias (2015) encuentran influyente sobre la salud aun controlando por factores sociales, puede aumentar la capacidad explicativa total del modelo que centra este trabajo.

Con este trabajo hemos delimitado el papel que desempeñan aquí el ocio, la salud física y mental, el apoyo y contacto con familia y amigos, aunque los resultados queden circunscritos a envejecientes de la Comunidad Valenciana, y sea necesario seguir estudiando esta cuestión en otras comunidades. Muy recientemente (Gañán y Villafruela, 2015) encuentran una gran disposición al ocio y tiempo libre, y por tanto a la mejora de la calidad de vida en mayores cursando en programas para adultos mayores en 
Castilla-León, lo que despierta el interés por poner a prueba el modelo explicativo aquí planteado y explorar matices en más contextos socioculturales españoles.

\section{Agradecimientos}

Proyecto subvencionado por Ministerio de Educación y Competitividad con referencia PSI2014-53280-R titulado "estudio longitudinal del envejecimiento exitoso en personas mayores en programas de aprendizaje a lo largo de la vida: impacto sobre el bienestar biopsicosocial". Los autores también agradecen al Vicerrectorado de Participación y Proyección Territorial de la Universitat de València su entusiasta apoyo a esta investigación.

\section{REFERENCIAS}

Alegria, M., Vila, D., Woo, M., Canino, G., Takeuchi, D., Vera, M., et al. (2009). Cultural relevance, and equivalence in the NLAAS instrument: integrating etic and emic in the development of cross-cultural measures for a psychiatric epidemiology and services study of Latinos. International Journal of Methods in Psychiatric Research, 13, 270288.

Arias, A.V., e Iglesias, S. (2015). La generatividad como una forma de envejecimiento exitoso. Estudio del efecto mediacional de los vínculos sociales. European Journal of Investigation in Health, Psychology and Education, 5(1), 109-120.

Baltes, P.B., y Baltes, M.M. (1990). Psychological perspectives on successful aging: The model of selective optimization with compensation. En P.B. Baltes y M.M. Baltes (Eds.): Successful aging: Perspectives from the behavioural sciences (pp. 1-35). Cambridge UK: Cambridge University Press.

Bentler, P.M. (1990). Comparative fit indices in structural models. Psychological Bulletin, 107, 238-246.

Brown, C.A., McGuire, F.A., y Voelkl, J. (2008). The link between successful aging and serious leisure. International Journal of Aging \& Human Development, 66, 73-95.

Chaves, M.L.; Camozzato, M.L.; Eizirik, C.L., y Kaye, J. (2009). Predictors of Normal and Successful Aging Among Urban-Dwelling Elderly Brazilians. The Journals of Gerontolology Series B: Psychological Sciences and Social Sciences, 64, 597-602.

Cosco, T.D., Prina, A.M., Perales, J., Stephan, B.C., y Brayne, C. (2013). Lay perspectives of successful ageing: a systematic review and meta-ethnography. BMJ Open, 3.

Cuenca, M. (2006). Aproximación multidisciplinar a los Estudios de Ocio. Documentos de Estudios de Ocio, 31. Bilbao: Universidad de Deusto.

Cuenca, M. (2012). Reflexiones sobre el ocio en el siglo XXI. Madrid: ARBOR-CSIC, 188, 754.

Diener, E., Emmons, R.A., Larsen, R.J., y Griffin, S. (1985). The satisfaction with life scale. Journal of Personality Assessment, 49, 71-75.

Fernández-Ballesteros, R., Zamarrón, M.D., López, M.D., Molina, M.A., Díez, J., Montero, P., et al. (2008). Envejecimiento con éxito: criterios y predictores. Psicothema, 22, 641-647.

Gañán, A., y Villafruela, I. (2015). El ocio, tiempo libre y calidad de vida para un envejecimiento activo, el caso de la Universidad de Burgos. European Journal of Investigation in Health, Psychology and Education, 5(1), 75-87. 
Hilton, J.M., Gonzalez, C.A., Saleh, M., Maitoza, R., y Cole, L. (2012). Perceptions of Successful Aging among Older Latinos in Cross-Cultural Context. Journal of Cross Cultural Gerontoly, 27, 183-199.

Hu, L., y Bentler, P.M. (1999). Cut-off criteria for fit indexes in covariance structure analysis: Conventional criteria versus new alternatives. Structural Equation Modeling, 6, 1-55

Iwamasa, G.Y., y Iwasaki, M.A. (2011). A new multidimensional model of successful aging: perceptions of Japanese American older adults. Journal of Cross Cultural Gerontology, 26, 261-78.

Karlin, N.J., Weil, J., Saratapun, N., Pupanead, S., y Kgosidialwa, K. (2014). Etic and Emic Perspectives on Aging Across Four Countries: Italy, Thailand, Botswana, and the United States. Ageing International, 39, 348-368.

Kahng, S.K. (2008). Overall successful aging: Its factorial structure and predictive factors. Asian Social Work and Policy Review, 2, 61-74.

Lazcano, I., Madariaga, A., y Doistua, J. (2010). El envejecimiento activo y su incidencia en la experiencia de ocio. ADOZ. Revista de Estudios de Ocio, 33, 117-147.

Lee, P., Lan, W., y Yen, T. (2011) Aging Successfully: A Four-Factor Model. Educational Gerontology, 37, 210-227.

Liang, J., y Luo, B. (2012) Toward a discourse shift in social gerontology: From successful aging to harmonious aging. Journal of Aging Studies, 26, 327-334.

Lubben, J., y Gironda, M. (2004). Measuring social networks and assessing their benefits. En C. Phillipson, G. Allan y D. Morgan (Eds.), Social networks and social exclusion: Sociological and policy perspectives (pp. 20-35). Hampshire, United Kingdom: Ashgate.

Phelan, E.A., Anderson, L.A., LaCroix, A.Z., y Larson, E.B. (2004). Older adults' views of "successful aging" - how do they compare with researchers' definitions? Journal of the American Geriatrics Society, 52, 211-216.

Pike, K.L. (1954). Language in relation to a unified theory of the structure of human behavior. Dallas, TX, US: Summer Institute of Linguistics.

Rowe, J.W. y Khan, R.L. (1987). Human aging: Usual and successful. Science, 237, 143-149.

Serrano, J.P., Latorre, J.M., y Gratz, M. (2014). Spain: promoting the welfare of older adults in the context of population aging. The Gerontologist, (first published online: March 14, 2014) doi:10.1093/geront/gnu010.

Steiger, J.H., y Lind, C. (1980). Statistically based tests for the number of common factors. Paper presented at the annual meeting of the Psychometric Society, Iowa City, IA

Ware, J.E., Snow, K.K., Kosinski, M., y Gandek, B. (1993). SF-36 Health Survey: manual and interpretation guide. Boston: New England Medical Center.

Ware, J.E., Kosinski, M., Dewey, J.E., y Gandek, B. (2001). How to Score and Interpret SingleItem Health Status Measures: A Manual for Users of the SF-8 Health Survey. Lincoln RI: QualityMetric Incorporated.

Recibido: 22 de abril de 2015

Recepción Modificaciones: 31 de octubre de 2015 Aceptado: 4 de noviembre de 2015 